\title{
El desarrollo y el aprendizaje del razonamiento causal: análisis de una tensión aparente*
}

\section{The Development and Learning of Causal Reasoning: Analysis of an Apparent Tension}

Recibido: junio 17 de 2013 | Revisado: mayo 29 de 2014 | Aceptado: junio 24 de 2014

\author{
WILLIAM JIMÉNEZ-LEAL ** \\ CHRISTIAN GAVIRIA \\ Universidad de los Andes, Bogotá, Colombia
}

doi.org/10.11144/Javeriana.UPSY13-4.darc

Para citar este artículo: Jiménez-Leal, W., \& Gaviria, C. (2014). El desarrollo y el aprendizaje del razonamiento causal: análisis de una tensión aparente. Universitas Psychologica, 13(4), 1603-1614. http://dx.doi.org/10.11144/Javeriana.UPSY13-4. darc

* William Jiménez-Leal fue financiado por el fondo FAPA de la Universidad de los Andes para la realización de esta investigación.

** Profesor Asistente. Departamento de Psicología. Correo electrónico: w.jimenezleal@uniandes.edu.co

\section{RESUMEN}

Como otras habilidades cognitivas, la capacidad de razonar causalmente cambia a través del ciclo vital. Sin embargo, no existe un acuerdo acerca de cómo ocurre su desarrollo. En este texto, se propone un análisis teórico particular para entender este proceso, a saber, la idea de que el razonamiento causal es una habilidad de dominio general que se va enriqueciendo con el refinamiento de habilidades metacognitivas que permiten desligar el razonamiento del contexto inmediato. Esta propuesta se basa en el análisis de evidencia de razonamiento causal en niños pequeños, así como de la integración de estas habilidades durante la adolescencia temprana, con procesos de argumentación y explicación. Se señalan además algunas diferencias metodológicas en los estudios con niños y jóvenes.

Palabras clave

razonamiento causal; causalidad; desarrollo cognitivo

\footnotetext{
A B S T R A C T

Like other cognitive skills, the ability to reason causally changes during the course of development from early childhood to adulthood. There is, however, no agreement about how its development occurs. In this paper we propose a theoretical analysis to understand this process, namely, the idea that causal reasoning is a domain-general ability that is gradually enriched by the refinement of metacognitive skills, which allows reasoning independently from the immediate context. This proposal is based on the analysis of evidence of causal reasoning in young children, as well as evidence of integration of these skills during early adolescence with processes of argumentation and explanation. The paper also points out some methodological differences in studies with children and adolescents.

Keywords

causal reasoning; causality; cognitive development
} 


\section{Introducción}

Nuestra habilidad para detectar y razonar con relaciones causales es fundamental para la vida diaria. Así, no sorprende que el concepto de causalidad, al igual que la amplia variedad de fenómenos asociados a él, ocupe un lugar central en muchas áreas de conocimiento psicológico: desde el estudio de la percepción y el aprendizaje, el razonamiento y el juicio, hasta los estudios comparativos del lenguaje, la cognición social y la metodología de la investigación (Alicke, Bloom, \& Rose, 2011; Glymour, 2001; Gopnik \& Wellman, 2012; Holyoak \& Cheng, 2011; Rips, 2011; Russo, 2008). No es sorprendente, entonces, que dicha centralidad se haga también manifiesta en el campo de la psicología del desarrollo.

Los trabajos de Piaget son, por supuesto, pioneros y referentes obligados al respecto. En el marco de sus ideas sobre epistemología genética y psicología del desarrollo, Piaget (1926/1987) propone que la idea de causalidad es parte de las nociones que se van afinando con el desarrollo cognitivo, pasando de un concepto centrado en el niño, de carácter animista y mágico, a una caracterización objetiva, ajustada a la realidad y en línea con la concepción científica. Así, en los primeros estadios del nivel sensoriomotor, los bebés ligan sus acciones a los fenómenos observados en el mundo: el ejemplo clásico es la observación del niño que al mover un cordón hala un móvil y posteriormente intenta mover el cordón para atraer otros objetos. Solo cuando los esquemas cognitivos relevantes se "descentran", las relaciones causales adquieren un carácter espacial y objetivo, primero, en relación con los objetos físicos con los que interactúan los niños y posteriormente con relaciones más abstractas. Piaget (1979) señala que el concepto de causalidad implícito en las acciones de los niños evoluciona desde una concepción a la Hume, donde a partir de la pura sucesión de fenómenos y la fuerza del hábito se infiere causalidad, pasando por la objetivación de las relaciones causales y llegando hasta la apreciación de que los vínculos causales objetivos solo pueden ser determinados a partir del razonamiento y el uso de los esquemas adecuados. Este es un proceso que tarda años, durante el cual los niños exhiben un tipo de pensamiento que es denominado por Piaget como precausal (1926/1987), caracterizado por acudir constantemente a principios de magia, animismo, artificialismo, transmisión por contacto y pensamiento supersticioso (e. g., "el mar fue hecho para bañarnos"; "Camilo tiene gripa, así que a mí también me va a dar porque me llamo igual"; "la luna está aquí porque quiere acompañarnos", etc.). Nótese que una de las marcas fundamentales del pensamiento precausal es la confusión entre la actividad psicológica y los mecanismos físicos relevantes, explicada por la mayor familiaridad del niño con su propia actividad (Piaget, 1926/1987).

La concepción piagetiana de la causalidad fue cuestionada fuertemente desde el momento mismo en que fue propuesta (Harris, 2009), pero es solo en los años 80 y 90 que se empieza a acumular evidencia sistemática de razonamiento causal eficaz y adecuado en niños pequeños (Kushnir \& Gopnik, 2005), la coexistencia de explicaciones de tipo mágico y causal mecanicista durante el desarrollo temprano (Rosengren, Kalish, Hickling, \& Gelman, 1994; Subbotsky, 2004) y la presencia fallos sistemáticos en el uso de principios de razonamiento proposicional en dominios causales con adultos (Shanks, 2004; Blanco, Mature, \& Vadillo, 2013). Todos estos elementos llevaron a replantear la manera en que se concibe la investigación sobre la noción de causalidad en psicología, no solo del desarrollo. En conjunto, estos hallazgos sugieren dos aspectos importantes de la concepción de causalidad a lo largo del desarrollo: la primera, el pensamiento mágico coexiste con el causal mecanicista a lo largo de la vida, si bien tiene primacía en la infancia temprana; la segunda, los niños pequeños son capaces de realizar inferencias causales complejas que anteriormente se creían solo aparecían en estadios tardíos del desarrollo. Estas dos características han implicado que la investigación sobre causalidad y desarrollo haya encontrado escenarios diversos en la psicología pospiagetiana.

El estudio psicológico de la comprensión causal se ha centrado en precisar cuáles son las claves que ofrecen información más efectiva sobre el entramado causal del mundo. Esta investigación depende de manera clave de análisis conceptuales previos 
(White, 1990) que privilegian uno u otro conjunto de claves. Así, por ejemplo, el énfasis temprano en el aprendizaje de contingencias por medio de procedimientos de condicionamiento clásico y operante, permitió privilegiar información referente a la contigüidad espacio-temporal de causas y efectos (Morris \& Hursch, 1982). Sin embargo, los esfuerzos centrados en destacar el papel de una clave a costa de las demás están condenados al fracaso. Existen muchas relaciones entre eventos, tales como covariación, orden temporal, contigüidad espacial y dependencia contrafáctica que si bien nos permiten aprender y hacer inferencias sobre relaciones causales, son condiciones suficientes, pero no necesarias para su aprendizaje (Einhorn \& Hogarth, 1986).

Las dificultades de la descripción psicológica están ligadas a los problemas en el análisis normativo del concepto de causalidad (Perales \& Shanks, 2003). La imposibilidad de brindar una definición formal de la causación, aceptada de forma amplia, se deriva directamente de la diversidad de significados que la afirmación "A causa B" connota en diferentes dominios de conocimiento (e. g., físico, biológico, psicológico, histórico, etc.), lo cual supone también la ausencia de un único estándar normativo respecto del cual se pueda calificar la corrección o incorrección de todos los juicios causales (Einhorn \& Hogarth, 1986).

Los retos que se presentan, tanto a nivel descriptivo como normativo, implican que cada concepción de la causalidad impone restricciones al rango de estrategias metodológicas que pueden ser empleadas en los estudios empíricos, en la medida en que seleccionan aquellas que son capaces de brindar más información sobre sus variables de interés. Por ejemplo, la tradición asociacionista, encarnada en el conductismo y el conexionismo, da prioridad a la sucesión regular de eventos y el descubrimiento de la contingencia potencial que existe entre tales elementos (Musca, Vadillo, Blanco, \& Matute, 2008). El punto clave aquí es que la comprensión causal emerge de procesos más básicos, como el de la detección de la regularidad estadística (Blanco et al., 2013). De otro lado, la idea de Piaget (1929) de la causación como agencia (e. g., capacidad para ejercer control sobre los eventos del entorno a partir de la propia acción) convierte a la entrevista en la técnica privilegiada para indagar si los niños se consideran o no agentes causales de fenómenos cotidianos, como la sucesión de día y noche. En contraste, los estudios clásicos de Albert Michotte (1962 citado en Gopnik, 2013) sobre la influencia del contacto físico, la organización temporal y la dirección del movimiento en la percepción de causalidad, no permiten explicar nexos causales más complejos, como los que naturalmente establecen las personas entre los deseos y las acciones de otros.

Así, es claro que el concepto mismo de causalidad empleado en investigación no es un constructo ateórico que permita su examen desde distintos puntos de vista. Por ejemplo, la relación entre agencia personal y causación también ha sido estudiada desde una perspectiva asociacionista, sin los compromisos teóricos de Piaget. Matute (1996) considera que la dimensión causal de la agencia individual es primitiva y, en ese sentido, tiende a ser sobreestimada. Desde este marco, se ha encontrado que la probabilidad de la consecuencia, con independencia de la contingencia real entre las acciones de los organismos y sus consecuencias, puede generar la ilusión de control (Blanco, Matute, \& Vadillo, 2011). Esta consiste en un sesgo de sobreestimación de la relación causal, que no ha sido explicado exitosamente, pero que parece estar determinado por la probabilidad marginal de ocurrencia del evento objetivo (Blanco et al., 2011; ver también, Yarritu, Matute \& Vadillo, 2014). Lo que resulta interesante para nuestro análisis es la diferencia en la explicación que puede establecerse desde ambas perspectivas: desde el análisis clásico piagetiano, la ilusión de control es evidencia de la tendencia al pensamiento mágico, mientras que desde el análisis de juicios de contingencia, la ilusión está guiada por la probabilidad objetiva de ocurrencia de un evento. Los términos en que se entiende cada explicación son, así, locales. Por ejemplo, un análisis reciente desde una perspectiva Bayesiana ha cuestionado que la sobrestimación de la agencia personal en relaciones causales se trate de un "sesgo" (Harris \& Osman, 2012). Sin embargo, esta crítica solo es válida si se acepta que la ilusión de control solo puede ser observada en condiciones 
de aprendizaje secuencial y con decisiones repetidas e independientes.

La carga teórica del investigador siempre determina qué datos se consideran más relevantes (Overton, 2006), y esto es especialmente cierto en campos de investigación que cubren un espectro muy amplio de fenómenos, como el de la causalidad. Antes de tratar de integrar los hallazgos provenientes de estas distintas aproximaciones, es necesario determinar si los procesos representacionales subyacentes a las inferencias causales son específicos de cada dominio de conocimiento o si, por el contrario, es posible identificar raíces comunes a todas ellas, a partir del estudio de su desarrollo a lo largo del tiempo.

Durante los últimas tres décadas, se han originado varias agendas de investigación sobre la comprensión causal, que han dado un mayor o menor énfasis al cambio de la comprensión causal durante el ciclo vital (Holyoak \& Cheng, 2011). El objetivo de este texto es contrastar dos de estas aproximaciones que se han centrado, por un lado, en las teorías intuitivas sobre el mundo físico y psicológico durante la infancia temprana y la edad preescolar (Baillargeon, Li, \& Yuan, 2009; Gopnik, 2013) y, por otra parte, en el desarrollo de formas complejas de razonamiento, pensamiento científico, argumentación y creencias epistemológicas durante la adolescencia y los inicios de la adultez (Barchfeld \& Sodian, 2009; Kuhn, 1991; Kuhn \& Pearsall, 2000). A continuación, se exploran algunos vínculos entre ambas líneas de investigación a partir del análisis de algunos de sus hallazgos más importantes. El objetivo es integrar los elementos de la comprensión causal que emergen de manera temprana durante el desarrollo y aquellos que surgen en un contexto de construcción y práctica en escenarios formales de aprendizaje. Dado que el propósito de este texto no es el de realizar una revisión exhaustiva, se examinarán solo algunos trabajos representativos de cada línea, con implicaciones para la discusión posterior de las interacciones entre aprendizaje y desarrollo a propósito de las inferencias causales. Nótese además que nos restringimos intencionalmente a trabajos que se encuentran alineados en la tradición de la psicología del desarrollo, si bien no se desconoce la relevancia desde trabajos de otras perspectivas en el estudio de los problemas presentados.

\section{Inferencias causales en la infancia temprana y la edad preescolar}

La pregunta inicial que debe abordar toda investigación del desarrollo del juicio causal es de naturaleza teórica: ¿Cuáles son las demandas de la tarea de inferir relaciones causales consistentes a partir de la experiencia? Asumiendo una perspectiva puramente asociacionista, los niños se enfrentan a situaciones donde hay eventos que se alternan en el tiempo con mayor o menor regularidad y donde, además, las propias acciones pueden modificar tales regularidades (Musca et al., 2008). Se pueden reconocer, entonces, dos fuentes principales de información del agente enfrentado a dicha tarea: en primer lugar, los patrones existentes en las contingencias o, dicho de otro modo, de las probabilidades condicionales de dos o más eventos repetibles. Segundo, la posibilidad de realizar intervenciones sistemáticas para explorar contingencias no conocidas y de registrar sus resultados. En el campo de la estadística y la inteligencia artificial, se han desarrollado sistemas formales capaces de inferir relaciones causales entre variables, a partir de probabilidades condicionales (Pearl, 2000). Desde un enfoque más psicológico, Glymour (2001) muestra que una amplia variedad de estructuras causales de conocimiento, propias del pensamiento adulto, pueden ser modeladas a partir de estos sistemas, llamados redes bayesianas. De acuerdo con este enfoque, la posibilidad y la calidad de la inferencia causal probabilística depende de la satisfacción de tres requerimientos principales: (1) capacidad del agente para detectar finas variaciones en la dependencia o independencia ${ }^{1}$ entre variables, a partir de sus probabilidades condicionales, (2) la actualización de las probabilidades previas a partir de nueva evidencia y (3) la coordinación de las probabilidades e intervenciones

1 Dos eventos A y B son independientes si la probabilidad de A por sí mismo es igual a la probabilidad condicional de A dado B, $P(A \mid B) P(A \mid B)$. En cualquier otro caso, A y B se consideran eventos relacionados. 
para refinar la estructura causal en construcción. Este análisis normativo se encuentra parcialmente anticipado en los análisis asociativos y basados en reglas de aprendizaje e inducción (Meder, Hagamayer, \& Waldmann, 2009; Perales \& Shanks, 2007). Sin embargo, la diferencia clave aquí es la incorporación de la noción de intervención que requiere la postulación de la representación de un modelo causal apropiado. A continuación, se revisarán algunos estudios recientes que dan cuenta del desarrollo temprano de estos prerrequisitos del pensamiento causal.

Utilizando un paradigma experimental de deshabituación como indicador de discriminación, Saffran, Aslin y Newport (1996) encontraron que niños de ocho meses son capaces de desempeñarse exitosamente en una tarea de segmentación de palabras, detectando las diferencias en las probabilidades de que un sonido siga a otro dentro de una misma palabra, en un pseudolenguaje creado por los investigadores. Estas regularidades fueron extraídas por los niños tras solo 2 minutos de exposición auditiva a las palabras del pseudolenguaje. En la misma línea, Kirkham, Sleemery y Johnson (2002) presentaron a niños de 2, 5 y 8 meses arreglos de estímulos visuales cuyo orden seguía ciertas regularidades estadísticas. Al presentar los estímulos en un orden que violaba esas regularidades, los niños de los tres grupos de edad mostraron ser igualmente sensibles al cambio. Estos hallazgos parecen apoyar la tesis de que la competencia para detectar patrones probabilísticos aparece en fases muy tempranas del desarrollo y no está ligada a un tipo particular de modalidad sensorial o contenido perceptual. No obstante, una limitación de este tipo de estudios es que no permiten establecer si los niños de estas edades son capaces de organizar dichas regularidades estadísticas en estructuras de conocimiento propiamente causales.

Realizar inferencias de causalidad supone capturar diferencias sutiles que pueden determinar la dependencia o independencia de eventos. Para examinar la manera en que los niños relacionan las diferencias entre probabilidades condicionales y atribución de causalidad, Gopnik, Sobel, Schulz y Glymour (2001) diseñaron una serie de experimen- tos con niños en edad preescolar, presentándoles diferentes secuencias de eventos y registrando las conclusiones que extraían a partir de ellas. Primero se les mostraba a los niños una máquina, diciéndoles que ciertos objetos, llamados "blickets", la activaban cuando se les colocaba sobre ella. Posteriormente, los niños veían si la máquina se activaba o no cuando dos objetos, A y B, se colocaban encima de ella juntos y por separado. La tarea de los niños consistía entonces en juzgar si A o B eran o no "blickets". Los investigadores variaron sistemáticamente el número de veces en que se hacía cada una de estas tres presentaciones (e. g., A, B, A y B juntos) junto con la activación contingente, o no, de la máquina. Los niños de 2 años y medio, al igual que los de 4 años, mostraron ser sensibles a la dependencia o independencia probabilística entre $A, B$ y la activación de la máquina, según fuese el caso. En los niños de 4 años, estos resultados fueron replicados en una tarea en un dominio de conocimiento distinto al físico, en la cual ellos debían determinar cuál era el color de las flores que hacían estornudar a un mono. Gopnik y sus colaboradores concluyen que estos hallazgos muestran cómo la noción de causalidad es un concepto a mitad de camino entre una idea de dominio específico (es necesario saber cómo operar en el dominio requerido para inferir causalidad) y de dominio general (pues claramente los niños son capaces de usar inputs con los que no tenían familiaridad en distintas tareas).

Un requerimiento crucial para las inferencias causales es también ser capaz de utilizar la información disponible sobre las probabilidades de los eventos para diseñar intervenciones que permitan recolectar información relevante. En el experimento de Gopnik et al. (2001), los niños de 3 y 4 años realizaron intervenciones específicas para observar relaciones no presentadas (como quitar uno de los objetos cuando la máquina estaba activada y observar si se apagaba o no). Schulz y Gopnik (2004) encontraron que niños de 4 años generaban nuevas formas de intervención consistentes con sus experiencias previas de dependencia entre eventos: después de que a los niños se les presentó una máquina que se encendía mediante una orden verbal ("Máquina, por favor enciende") 
y no por medio de su interruptor, se les preguntó cómo creían que se podía apagar la máquina. La mayoría de ellos respondió con la orden verbal contraria ("Máquina, por favor para"). Kushnir y Gopnik (2005) encontraron además que las acciones de los niños permiten no solo inferir la presencia de relaciones causales, sino también determinar qué tan fuertes son estas relaciones e incluso postular la existencia de causas encubiertas. En resumen, este conjunto de estudios muestra que niños, incluso de apenas 3 años, son capaces de determinar la estructura causal de una situación, a partir de información covariacional, y coordinar sus acciones con la estructura, de modo que se generen los efectos deseados (Schulz, Kushnir, \& Gopnik, 2007). En otras palabras, los niños son capaces de realizar intervenciones que, aunque similares, son cualitativamente distintas a las que realizan los adultos.

Una limitación de este tipo de investigaciones es que emplean arreglos experimentales no familiares que proveen únicamente indicadores asociativos de causalidad (i. e., covariación, contigüidad espacial y temporal) y aíslan artificialmente el efecto de otras formas de conocimiento que se relacionan naturalmente con los juicios causales. En consecuencia, estas aproximaciones tienen dificultades para explicar cómo los niños seleccionan los factores que ponen a prueba dentro de los modelos causales que elaboran y cómo asignan sus probabilidades iniciales, aspectos en los cuales las creencias previas, las metas de la tarea y la información disponible sobre mecanismos y explicaciones adquieren relevancia.

En esta línea, Tschirgi (1980) encuentra que niños de 7 a 11 años tienden a realizar intervenciones más controladas (i. e., manipular una variable mientras se mantienen otras constantes) cuando la meta es evitar un resultado indeseable e intervenciones menos controladas (i. e., manipular varias variables simultáneamente) que cuando se trata de producir un resultado positivo. Este hallazgo ilustra que, si bien los niños ya son competentes para identificar las probabilidades relevantes y realizar intervenciones informativas, la ejecución de acciones más o menos controladas está supeditada en parte al conocimiento previo y las metas de la tarea (Koslowsky \& Masnick, 2010).

Las acciones intencionales y sus efectos en el mundo también constituyen un insumo importante para las inferencias causales. Desde los trabajos piagetianos clásicos en esquemas de rodeo y coordinación de medios y fines (Piaget \& Inhelder, 1966), muchos estudios han encontrado consistentemente que entre el primer y segundo año de vida los niños distinguen entre la acción dirigida a metas de otros tipos de interacciones físicas, imitan las acciones de otros e incluso identifican las intenciones de terceros, a partir de la secuencia de sus propias acciones (Woodward \& Sommerville, 2000)². En adultos, la controlabilidad de los efectos tiende a ser sobreestimada sistemáticamente (Blanco et al., 2011), y puede ser responsable de creencias supersticiosas, por ejemplo.

La importancia de las acciones intencionales también puede ser incorporada en el marco de la inferencia causal probabilística, pues se puede explicar en términos de intervenciones realizadas, observadas o imaginadas. Como se señaló, la posibilidad de intervenir es uno de los requisitos necesarios para inferir causalidad de manera correcta, y al parecer esta capacidad es, no solo experimentada por los niños, sino que también es adecuadamente inferida en los contextos adecuados. Meltzoff, Waismeyer y Gopnik (2012) ofrecieron a dos grupos de niños un juego, similar al del blicket, con el cual no tenían familiaridad. En el grupo experimental, los niños observan las acciones de un adulto operando sobre el juguete con el objetivo de conseguir una canica, mientras que en el grupo control los niños simplemente observaron la operación del juguete que a veces producía la canica. Dado que los niños son capaces de reconocer acciones intencionales, Meltzoff et al. (2012, Experimento 1), efectivamente, encontraron que los niños usaron esta información para inferir adecuadamente cómo producir una canica de manera más efectiva en el grupo experimental que en el grupo control.

2 Sin embargo, las acciones intencionales no tienen una distribución probabilística y parecen tener un estatus especial. 
La evidencia examinada sugiere que, para el inicio de la edad escolar, los "bloques de construcción” de las inferencias causales ya están presentes; el niño puede identificar regularidades, realizar intervenciones sistemáticas e inferencias causales relativamente complejas, así como reconocer y aprender a partir de intervenciones observadas. ¿Qué ocurre con estas habilidades en fases posteriores del desarrollo? A continuación, se examinarán brevemente algunos trabajos que dan respuesta a este interrogante.

\section{Inferencias causales en la adolescencia y la adultez}

Una diferencia fundamental entre las investigaciones con niños y los estudios con adolescentes es que, para estas últimas, la naturaleza de las tareas utilizadas es predominantemente verbal. Las tareas utilizadas en las investigaciones presentadas en la sección anterior, se centran en predicción e intervención causal, mientras que los estudios realizados con adolescentes privilegian tareas que involucran posdicción (e. g., explicación) y argumentación. Los estudios acerca del origen del pensamiento científico son un buen ejemplo al respecto. Kuhn y Pease (2006) compararon el desempeño de niños de entre 11 y 12 años con el de estudiantes universitarios, en una misma tarea de razonamiento causal. A los participantes se les decía que una organización caritativa pensaba regalar un oso de felpa a sus donantes y que debían decidir qué accesorios (de un conjunto de 7) podían incluirse, con el fin de que se incrementaran las donaciones. Después de presentarles datos que relacionaban la presencia de ciertos conjuntos de accesorios con incrementos en las donaciones, se les pedía que trataran de explicar estos datos y que juzgaran cuáles accesorios ayudarían a incrementar los recaudos. Se encontró que la corrección de los juicios, entendida como reconocimiento de la covariación, estaba correlacionada positivamente con la edad, aunque la variabilidad entre sujetos dentro de cada grupo fue considerable: mientras que hubo muchos niños que se desempeñaron tan bien como la mayoría de los adultos, hubo adultos que no lo hicieron mejor que la mayoría de los niños. Un aspecto clave de este estudio es que el contenido de la tarea (e. g., relaciones causales entre accesorios de muñecos de felpa y donaciones) no resultaba familiar para ninguno de los dos grupos de edad, de modo que las diferencias entre grupos no podían ser atribuidas a experiencia o experticia de dominio. Estos resultados coinciden con la literatura que muestra un alto grado de ajuste entre los juicios causales adultos y ciertos modelos normativos probabilísticos, con excepción de ciertas desviaciones sistemáticamente observadas (Shanks, 2004).

Por otro lado, en un estudio realizado con una muestra heterogénea de participantes (40 usuarios del metro de Nueva York con edades entre los 16 y 50 años o más), Kuhn (2007) encontró resultados muy diferentes. A la manera del estudio anterior, a los participantes se les presentaba información sobre la presencia o ausencia de varios factores (premio a la entrada, comedia, subasta) y el éxito en los recaudos de una fiesta benéfica. Aunque los datos presentados solo permitían asegurar la dependencia entre un único factor (subasta) y los recaudos, el $83 \%$ de los participantes también estableció relaciones entre las donaciones y otros factores. En una segunda tarea, se les pedía a las personas predecir si los recaudos serían altos en futuras fiestas que tuvieran o no los factores que ellos mismos habían especificado. Se encontró que los participantes hicieron predicciones inconsistentes con sus atribuciones causales previas al incluir factores que antes no habían considerado como causalmente relacionados con un mayor o menor recaudo de fondos en las fiestas. Estos hallazgos son consistentes con los de literatura sobre prueba de hipótesis y sesgo de confirmación (Sloman \& Fernbach, 2008), la cual sugiere que las personas tienden a dar más peso a la evidencia positiva de covariación entre dos variables que a la de evidencia negativa. En algunos casos, este sesgo en la evaluación de la evidencia puede llevar a sobreestimar la influencia causal de una variable sobre otra.

A diferencia de los resultados en el desarrollo temprano de los componentes básicos para la inferencia causal (Gopnik, 2013), los estudios sobre la competencia causal en adultos parecen proporcio- 
nar una imagen ambigua: por un lado, estudios de laboratorio que evidencian sensibilidad a las estructuras causales de eventos discretos y a la magnitud de sus parámetros; por otro, el fracaso en tareas sencillas de razonamiento causal con contenidos cotidianos debido a las creencias y expectativas previas. ¿Cómo se explica esta discrepancia?

Existen dos diferencias fundamentales entre los estudios de Kuhn y Pease (2006) y Kuhn (2007) que pueden arrojar luces sobre esta pregunta: las características de la muestra y los contenidos de la tarea. Mientras que la mayoría de los estudios en razonamiento causal se llevan a cabo con estudiantes en ambientes académicos, Kuhn reunió una muestra con un rango de edad mucho mayor y, probablemente, también de formación educativa. Adicionalmente, los materiales utilizados en estos estudios conllevan una restricción artificial y no siempre explícita: el participante debe ceñirse a la información proporcionada en la tarea a la hora de hacer sus inferencias, ignorando el conocimiento previo que pueda tener sobre el tema de la misma. Si bien estas restricciones se asumen comúnmente en ambientes de educación formal, resultan contraintuitivas en la vida cotidiana: en principio, no hay razón para que uno deba concentrarse solo en el segmento más inmediato de su experiencia al formar una opinión o tomar una decisión importante. Sin embargo, el poder extraer inferencias a partir de una pieza aislada de información y ser capaz de diferenciar en qué contextos se requiere o no integrar los conocimientos previos a la inferencia, son también habilidades indispensables en muchos entornos de trabajo colaborativo. Es probable que los participantes no familiarizados con entornos académicos incorporen en la tarea información proveniente de sus experiencias previas sobre la dependencia entre variables, sin que esto implique que carezcan de la competencia para realizar inferencias causales. Esta disociación entre lo que las personas pueden hacer (competencia) y lo que efectivamente hacen (actuación) en función de los contenidos con los que interactúan es un hallazgo común en la investigación del razonamiento deductivo, cuyas tareas demandan concentrarse exclusivamente en la forma (Evans, 2010; Evans, Barston, \& Pollard,
1983). Si existe esta disociación, se esperaría que los contenidos menos familiares atenúen la interferencia de las experiencias previas en los juicios causales, como efectivamente sucede en el estudio de Kuhn y Pease (2006) y en estudios de laboratorio con estudiantes universitarios (Shanks, 2004; Sloman \& Fernbach, 2008).

En este contexto, Kuhn, Katz y Dean (2004) postulan que los sistemas inferenciales adultos están compuestos por una amplia gama de reglas más o menos sofisticadas que se emplean para interpretar el estatus causal de múltiples variables a la luz de distintas evidencias y que se pueden aplicar selectivamente en función del contexto. Por supuesto, la variabilidad entre individuos a propósito de este repertorio de reglas dependerá de factores tales como la experticia y el tipo de instrucción recibida. Mientras que algunos individuos (e. g., estudiantes universitarios) en ciertos casos (e. g., experimentos de laboratorio con materiales no familiares) pueden identificar covariaciones y realizar intervenciones de manera consistente con modelos de redes bayesianas, en otros contextos las inferencias a propósito de qué factores son causales con respecto a un cierto efecto dependerán en mayor medida de las expectativas generadas por el conocimiento previo sobre la estructura causal de los fenómenos y de habilidades metacognitivas. Así, varios estudios han encontrado que la facilidad para imaginar o explicar una hipótesis causal consistente con el conocimiento previo se asocia a un aumento en la confianza en su veracidad (Koehler, 1991). En el mismo sentido, Lombrozo (2007, Experimento 3) encontró que estudiantes universitarios que eligen una explicación simple aunque improbable tienden a sobreestimar la ocurrencia del factor causal correspondiente, en comparación con aquellos que prefieren una explicación más compleja. La interacción entre indicadores asociativos y conocimiento previo sobre mecanismos y explicaciones es pues un instrumento de "doble filo": si bien puede ayudar a distinguir entre relaciones de covariación relevantes e irrelevantes (e. g., la existencia de un mecanismo puede sugerir que cierta covariación aparentemente extraña es causal, mientras que cierta covariación sistemática puede sugerir la existencia de un meca- 
nismo subyacente), también puede provocar que se realicen inferencias sesgadas a partir de datos confusos e intervenciones poco controladas (Koslowski \& Masnick, 2010).

\section{Conclusiones: inferencias causales y el desarrollo del aprendizaje}

Como se ha mostrado, en las inferencias causales adultas entran en juego dos tipos de competencias que, aunque pueden servir a fines comunes, son de naturaleza distinta: una, de dominio general, que permite la identificación de relaciones probabilísticas condicionales entre eventos y su actualización en función de los resultados de intervenciones sistemáticas; otra, de carácter metacognitivo y altamente sensible al contexto, que hace posible la representación de nueva información disociada del dominio y del conocimiento previo. Mientras que la primera surge tempranamente en la niñez, la segunda no aparece espontáneamente en algún punto específico del desarrollo. La variabilidad encontrada por Kuhn y Pease (2006) entre los desempeños de niños y adultos a propósito de una misma tarea de razonamiento causal puede ser un indicador de que en muchos casos la edad no es el factor clave, sino la experiencia de cada individuo en tareas que le demandan la actualización de ambas competencias. En la misma línea, Stanovich y West (2000) han encontrado sistemáticamente que los estilos de pensamiento, más que el desarrollo de las habilidades cognitivas tradicionalmente medidas por las pruebas de inteligencia, se asocian con la capacidad para ejercer control metacognitivo y la reducción de sesgos inferenciales en tareas de razonamiento probabilístico.

En esta línea, no sorprende la ausencia de estudios sobre neurociencias que tomen la maduración como proceso clave para el razonamiento causal. Si bien ha habido intentos sistemáticos de investigar el sustrato neural del razonamiento deductivo (por ejemplo, Goel, 2007), este no es el caso para el caso del razonamiento causal. La gran excepción es el trabajo de Patterson y Barbey (2013) que investigan correlatos neuronales de desempeño, utilizando teorías recientes cognitivas sobre razonamiento causal, pero solo a propósito de la capacidad de dominio general. Al respecto se deben notar dos cosas: la primera, su propuesta de un marco desde la neurociencia es subsidiaria de teorías de razonamiento en adultos, que no se ocupan del problema del desarrollo. La segunda es que se trata todavía de un examen con categorías gruesas, donde se identifica activación generalizada de grandes áreas como la corteza sensoriomotora, por ejemplo. El trabajo de Patterson y Barbey es un esfuerzo pionero de integración entre la investigación en neurociencia y la psicología del razonamiento causal, pero limitado por los conceptos mismos usados en las teorías cognitivas respectivas.

Los hallazgos aquí descritos tienen implicaciones en al menos tres ámbitos diferentes: la investigación básica en razonamiento causal, la medición de las competencias cognitivas y la toma de decisiones en el diseño curricular. En primer lugar, la mayoría de estudios experimentales en psicología del pensamiento se realizan con estudiantes universitarios, bajo el supuesto de que la naturaleza básica de tales procesos permite hacer generalizaciones plausibles de sus resultados a otros segmentos de población. No obstante, la variabilidad entre participantes, encontrada en los trabajos de Deanna Kuhn y sus colaboradores (Kuhn, 2004, Kuhn et al. 2007), restringe el alcance de estas generalizaciones y demanda un control cuidadoso de las diferencias individuales en los entornos de aprendizaje a los que han sido expuestos, a fin de preservar la validez ecológica de los resultados. Por su parte, la evidencia de la disociación en el desarrollo de competencias cognitivas y metacognitivas a propósito de la inferencia causal sugeriría que los instrumentos de medición de aptitudes e inteligencia no dan cuenta de la totalidad de capacidades requeridas para realizar inferencias en la vida cotidiana, lo que podría poner en cuestión su uso extendido como predictor del desempeño posterior en ambientes reales (Stanovich, 2009).

Finalmente, estos hallazgos hacen pensar que los objetivos educativos no deben limitarse a la enseñanza explícita de reglas formales que refinen habilidades de dominio general ya provistas por el desarrollo, sino más bien favorecer la comprensión de lo que significa involucrarse en actividades 
inferenciales que demandan monitoreo continuo y formas complejas de control metacognitivo. En la medida en que esta comprensión es sensible a la instrucción y emerge en la interacción con entornos enriquecidos de aprendizaje (Siler \& Klahr, 2012), esta experiencia puede hacer la diferencia en la adquisición de la competencia para razonar adecuadamente sobre causas y efectos. En este sentido, es posible afirmar que adquirir conocimiento declarativo sobre la estructura causal del mundo-y no solo adquirir habilidades para razonar causalmente- también hace parte del desarrollo cognitivo (Koslowski \& Masnick, 2010). Siegler (2000) señala que las distinciones entre aprendizaje y desarrollo son puramente instrumentales, en la medida en que ambas hacen parte de un único proceso. La inferencia causal es un caso paradigmático de cómo desarrollo y aprendizaje tiene patrones distintivos de evolución temporal, sin que esto les impida integrarse en totalidades funcionales durante el ciclo vital de cada individuo. Comprender cómo llegan a coordinarse ambos procesos a lo largo del tiempo sigue siendo una de las grandes preguntas de la psicología del desarrollo cognitivo.

\section{Referencias}

Alicke, M. D., Bloom, D., \& Rose, D. (2011). Causation, norm violation, and culpable control. Journal of Philosophy, 108(12), 670-696.

Baillargeon, R., Li, J., Ng., W., \& Yuan, S. (2009). An account of infants' physical reasoning. En A. Woodward \& A. Needham (Eds.), Learning and the infant mind (pp. 66-116). New York: Oxford University Press.

Barchfeld, P., \& Sodian, B. (2009). Differentiating theories from evidence: The development of argument evaluation abilities in adolescence and early adulthood. Informal Logic, 29(4), 396-416.

Blanco, F., Matute, H., \& Vadillo, M. A. (2011). Making the uncontrollable seem controllable: The role of action in the illusion of control. Quarterly Journal of Experimental Psychology, 64(7), 1290-1304.

Blanco, F., Matute, H., \& Vadillo, M. A. (2013). Interactive effects of the probability of the cue and the probability of the outcome on the overestimation of null contingency. Learning $\mathcal{E}$ Behavior, 41(4), 333-340.

Einhorn, H. J. \& Hogarth, R. (1986). Judging probable cause. Psychological Bulletin, 99(1), 3-19.

Evans, J. St. B. (2010). Thinking twice: Two minds in one brain. New York: Oxford University Press.

Evans, J. St. B., Barston, J. L., \& Pollard, P. (1983). On the conflict between logic and belief in syllogistic reasoning. Memory and Cognition, 11(3), 285-306.

Goel, V. (2007). Anatomy of deductive reasoning. Trends in Cognitive Sciences, 11(10), 435-441.

Glymour, C. (2001). The mind's arrows: Bayes nets and graphical causal models in psychology. Cambridge: MIT Press.

Gopnik, A. (2013). Causality. En P. Zelazo (Ed.), The oxford handbook of developmental psychology. New York: Oxford University Press.

Gopnik, A., Sobel, D. M., Schulz, L., \& Glymour, C. (2001). Causal learning mechanisms in very young children: two-, three-, and four-year-olds infer causal relations from patterns of variation and covariation. Developmental Psychology, 37(5), 620-629.

Gopnik, A., \& Wellman, H. M. (2012). Reconstructing constructivism: Causal models, Bayesian learning mechanisms, and the theory theory. Psychological Bulletin, 138(6), 1085-108.

Harris, P. L. (2009). Piaget on causality: The Whig interpretation of cognitive development. British Journal of Psychology, 100(Pt 1 A), 229-232.

Harris, A. J. L., \& Osman, M. (2012). The illusion of control: A Bayesian perspective. Synthese, 189(Supl. 1), 29-38.

Holyoak, K. J., \& Cheng, P. W. (2011). Causal learning and inference as a rational process: The new synthesis. Annual Review of Psychology, 62, 135-163.

Kirkham, N., Sleemer, J., \& Johnson, S. (2002). Visual statistical learning in infancy: Evidence for a domain general learning mechanisms. Cognition, 83, B4135-B142.

Koehler, D. J. (1991) Explanation, imagination and confidence in judgment. Psychological Bulletin, 110(3), 499-519.

Koslowski, B., \& Masnick, A. (2010). Causal reasoning and explanation. En U. Goswami (Ed.), Handbook of child cognitive development (2.a ed., pp. 377-398). Oxford: Blackwell Publishers. 
Kuhn, D. (1991). The skills of argument. Cambridge: Cambridge University Press.

Kuhn, D. (2007). Jumping to conclusions: Can people be counted on to make sound judgments? Scientific American Mind, 18(1), 44-51.

Kuhn, D., Katz, J. B., \& Dean, D. (2004). Developing reason. Thinking and Reasoning, 10(2), 197-219.

Kuhn, D., \& Pearsall, S. (2000). Developmental origins of scientific thinking. Journal of Cognition Development, 1, 113-129.

Kuhn, D., \& Pease, M. (2006). Do children and adults learn differently? Journal of Cognition and Development, 7(3), 279-293.

Kushnir, T., \& Gopnik, A. (2005). Young children infer causal strength from probabilities and interventions. Psychological Science, 16(9), 678-683.

Lombrozo, T. (2007) Simplicity and probability in causal explanation. Cognitive Psychology, 55(3), 232-257.

Matute, H. (1996). Illusion of control: Detecting response-outcome independence in analytic but not in naturalistic conditions. Psychological Science, 7(5), 289-293.

Meder, B., Hagmayer, Y., \& Waldmann, M. R. (2009). The role of learning data in causal reasoning about observations and interventions. Memory $\mathcal{E}$ Cognition, 37(3), 249-64.

Meltzoff, A. N., Waismeyer, A., \& Gopnik, A. (2012). Learning about causes from people: Observational causal learning in 24-month-old infants. Developmental Psychology, 48(5), 1215-1228.

Morris, E. K., \& Hursch, D. E. (1982). Behavior analysis and developmental psychology: Metatheoretical considerations. Human Development, 25, 344-349.

Musca, S. C., Vadillo, M. A., Blanco, F., \& Matute, H. (2008). Associative and connectionist accounts of biased contingency detection in humans. En R. M. French \& E. Thomas (Eds.), From associations to rules: Connectionist models of behavior and cognition (pp. 16-27). Singapore: World Scientific.

Overton, W. F. (2006). Developmental psychology: Philosophy, concepts, methodology. En W. Damon \& R. M. Lerner (Eds. de la serie) \& R. M. Lerner (Ed. del volumen). Handbook on child psychology: Vol. 1. Theoretical models of human development (6.a ed., pp. 953-257). Hoboken, NJ: Wiley.
Patterson, R., \& Barbey, A. K. (2013). A cognitive neuroscience framework for causal reasoning. En J. Grafman \& F. Krueger (Eds.), The neural representation of belief systems, pp. 77 - 120. Hove, UK: Psychology Press.

Pearl, J. (2000). Causality: Models, reasoning, and inference. Cambridge, UK: Cambridge University Press.

Perales, J. C., \& Shanks, D. R. (2003). Normative and descriptive accounts of the influence of power and contingency on causal judgement. The Quarterly Journal of Experimental Psychology, 56(6), 977-1007.

Perales, J. C., \& Shanks, D. R. (2007). Models of covariation-based causal judgment: A review and synthesis. Psychonomic Bulletin E⿱ Review, 14(4), 577-596.

Piaget, J. (1987). La representación del mundo en el niño. Madrid: Morata. (Trabajo original publicado en 1926)

Piaget, J., \& Inhelder, B. (1966). Psicología del niño. Madrid: Morata.

Piaget, J. (1979). Tratado de lógica y conocimiento científico (Vol. 4). Buenos Aires: Paidós.

Rips, L. J. (2011). Causation from perception. Perspectives on Psychological Science, 6(1), 77-97.

Rosengren, K. S., Kalish, C. W., Hickling, A. K., \& Gelman, S. A. (1994). Exploring the relation between preschool children's magical beliefs and causal thinking. British Journal of Developmental Psychology, 12(1), 69-82.

Russo, F. (2008). Causality and causal modelling in the social sciences: measuring variations. Dordrecht: Springer.

Saffran, J. R., Aslin, R. N., \& Newport, E. L. (1996). Statistical learning by 8 -month old infants. Science, 274(5294), 1926-1928.

Schulz, L., \& Gopnik, A. (2004). Causal learning across domains. Developmental Psychology, 40 (2), 162-176.

Schulz, L., Kushnir, T., \& Gopnik. A. (2007). Learning from doing: Intervention and causal inference in children. En A. Gopnik \& L. Schulz (Eds.), Causal learning: Psychology, philosophy, computation (pp. 67-85). New York: Oxford University Press.

Shanks, D. (2004). Judging covariation and causation. En D. J. Koehler \& N. Harvey. (Eds.), Blackwell handbook of judgment and decision making (pp. 220239). Oxford: Blackwell. 
Siegler, R. S. (2000). The rebirth of children's learning. Child Development, 71(1), 26-35.

Siler, S. \& Klahr, D. (2012). Detecting, classifying, and remediating children's explicit and implicit misconceptions about experimental design. En R. W. Proctor \& E. J. Capaldi (Eds.), Psychology of science: Implicit and explicit processes. New York: OUP.

Sloman, S. A., \& Fernbach, P. M. (2008). The value of rational analysis: An assessment of causal reasoning and learning. En N. Chater \& M. Oaksford (Eds.), The probabilistic mind: Prospects for rational models of cognition (pp. 481-495). Oxford: OUP.

Stanovich, K. E. (2009). What intelligence tests miss: The psychology of rational thought. New Haven: Yale University Press.

Stanovich, K. E., \& West, R. F. (2000). Individual differences in reasoning: Implications for the ratio- nality debate? Behavioral and Brain Sciences, 23(5), 645-665.

Subbotsky, E. (2004). Magical thinking in judgments of causation: Can anomalous phenomena affect ontological causal beliefs in children and adults? British Journal of Developmental Psychology, 22(1), 123-152.

Tschirgi, J. E. (1980). Sensible reasoning: A hypothesis about hypotheses. Child Development, 51(1), 1-10.

Yarritu, I., Matute, H., \& Vadillo, M. A. (2014). Illusion of control: The role of personal involvement. Experimental Psychology, 61(1), 38-47.

White, P. (1990). Ideas about causation in philosophy and psychology. Psychological Bulletin, 108(1), 3-18.

Woodward, A., \& Sommerville, J. (2000). Twelvemonth-old infants interpret action in context. Psychological Science, 11(1), 73-77. 